\title{
Prognostic Relevance of Liver Stiffness Assessed by Transient Elastography in Patients With Acute Decompensated Heart Failure
}

\author{
Yuki Saito, MD, PhD; Mahoto Kato, MD; Koichi Nagashima, MD, PhD; \\ Koyuru Monno, MD; Yoshihiro Aizawa, MD, PhD; Yasuo Okumura, MD, PhD; \\ Naoki Matsumoto, MD, PhD; Mitsuhiko Moriyama, MD, PhD; Atsushi Hirayama, MD, PhD
}

\begin{abstract}
Background: Acute decompensated heart failure (ADHF) is often accompanied by liver congestion through increased right atrial pressure (RAP). Liver stiffness (LS) assessed non-invasively using transient elastography is related to increased RAP and liver congestion in patients with general HF. We investigated the relationship of LS with clinical and echocardiographic variables and outcomes in patients with ADHF.

Methods and Results: The subjects were 105 patients with ADHF admitted to hospital between October 2016 and June 2017. Patients were divided into 2 groups based on median LS at admission (low LS <8.8 kPa [n=52] vs. high LS $\geq 8.8 \mathrm{kPa}$ [ $=53$ ]). Death from cardiovascular disease and readmission for HF were primary endpoints. Total bilirubin and $\gamma$-glutamyl transpeptidase levels, MELD-XI score, diameters of the inferior vena cava and right ventricle, and severity of tricuspid regurgitation were greater in the high LS group (all $\mathrm{P}<0.05$ ). During a median (interquartile range) follow-up period of 153 (83-231) days, cardiac events occurred in 29 patients $(54 \%)$ in the high LS group and in $13(25 \%)$ in the low LS group ( $P=0.001)$. After adjusting for variables that influence organ congestion, a high LS $\geq 8.8 \mathrm{kPa}$ was still significantly associated with cardiac events (all $\mathrm{P}<0.05)$.
\end{abstract}

Conclusions: Increased LS measured by transient elastography reflects RAP elevation, hepatic congestion, and hepatic dysfunction. LS upon admission may be a useful prognostic marker in patients with ADHF.

Key Words: Echocardiography; Heart failure; Liver stiffness

A cute decompensated heart failure (ADHF) is often accompanied by hepatic congestion through the increased right atrial pressure (RAP). ${ }^{1}$ Hepatic dysfunction caused by hepatic congestion is referred to as congestive hepatopathy and is related to an adverse outcome. 2,3 Transient elastography (TE) is a reliable, noninvasive technology that can reproducibly assess liver stiffness (LS) by measuring the liver shear wave velocity. LS is a useful for evaluation of the severity of liver fibrosis in chronic viral hepatitis and other chronic liver conditions. $^{\mathbf{4 , 5}}$ It has recently been reported that liver congestion also leads to increased LS because anatomically the liver is wrapped by a non-elastic capsule. ${ }^{6}$ Therefore, LS assessed by TE could be a marker of increased RAP and liver congestion in patients with general heart failure (HF). ${ }^{7}$ However, the effect of LS on the clinical variables and prognosis of patients with ADHF remains unclear. Therefore, the aim of this study was to investigate the association of LS with clinical and echocardiographic variables and prognosis in patients with ADHF.

\begin{abstract}
Methods
Patients and Protocol

A total of 154 patients with ADHF who were admitted to Nihon University Itabashi Hospital between October 2016 and June 2017 were prospectively enrolled in the study. The Framingham criteria were used to diagnose ADHF. ${ }^{8}$ Patients with excessive alcohol intake $(>20 \mathrm{~g} /$ day $)(n=7)$, acute coronary syndrome $(n=5)$, viral hepatitis $(n=2)$, hepatic tumors $(n=2)$, body mass index $>30 \mathrm{~kg} / \mathrm{m}^{2}(\mathrm{n}=11)$, an invalid LS evaluation $(n=10)$, or lost to follow-up $(n=12)$ were excluded, leaving 105 subjects in the study. LS was measured within 2 days of admission and patients were separated into 2 groups based on the median value $(8.8 \mathrm{kPa})$. According to the Clinical Scenarios (CS) classification, systolic blood pressure (SBP) at admission $>140 \mathrm{mmHg}$ is defined as vascular failure pattern onset (CS1). ${ }^{9}$ SBP at admission $100-140 \mathrm{mmHg}$ is defined as cardiorenal failure pattern (CS2) and SBP at admission $<100 \mathrm{mmHg}$ is defined as cardiac failure pattern $(\mathrm{CS} 3) .^{9}$
\end{abstract}

Received December 6, 2017; revised manuscript received February 24, 2018; accepted February 26, 2018; released online April 17, 2018 Time for primary review: 33 days

Division of Cardiology (Y.S., M.K., K.N., K.M., Y.A., Y.O., A.H.), Division of Gastroenterology and Hepatology (N.M., M.M.), Department of Medicine, Nihon University School of Medicine, Tokyo, Japan

Mailing address: Yuki Saito, MD, PhD, Division of Cardiology, Department of Medicine, Nihon University School of Medicine, 30-1 Ohyaguchi-kamicho, Itabashi-ku, Tokyo 173-8610, Japan. E-mail: yuukimoriya310@yahoo.co.jp

ISSN-1346-9843 All rights are reserved to the Japanese Circulation Society. For permissions, please e-mail: cj@j-circ.or.jp 


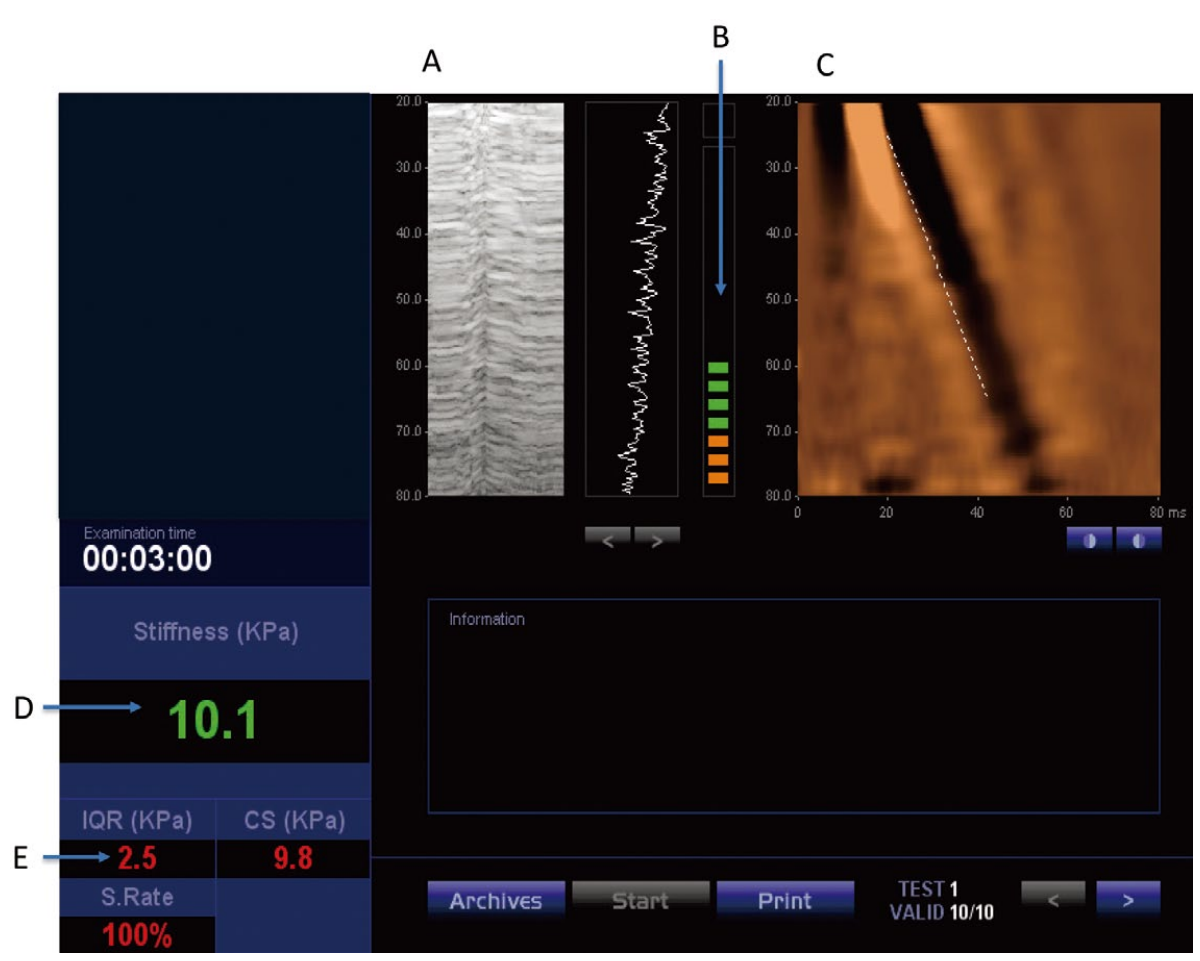

Figure 1. Representative example of liver stiffness (LS) measurement. (A) Premeasurement $M$ and $A$ mode ultrasound display of the liver. (B) Indicator of pressure applied to the patient's skin. (C) Post-measurement shear wave propagation image. The Y-axis displays the depth from the probe and the $\mathrm{X}$-axis displays the propagation time. Therefore, the oblique dotted line indicates the velocity of shear wave propagation. Stiffness is calculated from this velocity. (D) Median LS of 10 valid measurements. (E) Interquartile range of LS measurements. In this case, the median LS of 10 valid measurements was $10.1 \mathrm{kPa}$.

The study protocol and use of patient information was approved by the Nihon University Itabashi Hospital's Ethics Committee, and informed consent was given by all patients. Demographic information was obtained from the medical records.

\section{Measurement of LS Using TE}

LS measurement was performed by TE (FibroScan; Echosens, Paris, France), as described previously. ${ }^{10} \mathrm{~A}$ representative example of this measurement is shown in Figure 1. An experienced cardiologist blinded to the clinical data performed the procedure. With the patient in a fasted state and lying supine with the right arm at maximal abduction, the operator placed the probe perpendicularly over the right intercostal space. For each patient, 10 valid measurements of LS (in kilopascals $(\mathrm{kPa})$ ) were obtained and the median value was determined. The evaluation was considered reliable if the success rate was at least $60 \%$ and the interquartile range to median ratio was $<30 \%$.

\section{Echocardiography}

Experienced sonographers who were blinded to all other data performed echocardiography on the same day of LS measurement. A Vivid 7 cardiovascular ultrasound system (GE Vingmed Ultrasound A.S., Horten, Norway) was used with the patient in a supine position. Echocardiographic parameters were obtained using American Society of Echocardiography guidelines. ${ }^{\mathbf{1 1} 12}$ Briefly, the left ventricular ejection fraction (LVEF) was measured by the modified
Simpson method. The LV end-diastolic diameter (LVDd), LV end-systolic diameter (LVDs) and left atrial diameter (LAd) were obtained from the parasternal long-axis view. The peak early diastolic (E) and late diastolic (A) velocities were measured from transmitral flow velocity curves. The early diastolic (e') and late diastolic (a') myocardial velocities were obtained from tissue Doppler imaging of the mitral annulus at the septal position. The right ventricular end-diastolic diameter (RVDd) and tricuspid annular plane systolic excursion (TAPSE) were measured from a RVfocused apical 4-chamber view. Mitral regurgitation and tricuspid regurgitation (TR) were graded on a 4-point scale based on color-flow Doppler images. Measurement of the TR jet area was done on color Doppler images of TR flow. From the subcostal view, the diameter of the inferior vena cava (IVC) in its long axis was measured within $3 \mathrm{~cm}$ of the IVC-RA junction during passive respiration. Estimated RAP was calculated from the diameter of the IVC and respiratory changes, based on the guidelines. ${ }^{\mathbf{1 2}}$

\section{Evaluation of Liver Function}

Routine blood tests were performed on admission. The composite Model for End-Stage Liver Disease excluding INR (MELD-XI) score was calculated for evaluation of liver function: a high score relates to an adverse clinical outcome in patients with HF. ${ }^{13}$ The MELD-XI score was calculated as follows: [5.116 log (total bilirubin)+11.766 $\log$ (creatinine)+9.44], with scores $<1$ given a value of $1 . .^{13}$ 
Table 1. ADHF Patients' Characteristics and Laboratory Data Stratified by Median LS Value

\begin{tabular}{|c|c|c|c|}
\hline & $\begin{array}{c}\text { Low LS (<median) } \\
\qquad(n=52)\end{array}$ & $\begin{array}{c}\text { High LS ( } \geq \text { median) } \\
(n=53)\end{array}$ & $P$ value \\
\hline \multicolumn{4}{|l|}{ Baseline clinical data } \\
\hline Age, years & $80(71-85)$ & $77(64-84)$ & 0.30 \\
\hline Male, n (\%) & $37(71)$ & $39(73)$ & 0.78 \\
\hline Body mass index, $\mathrm{kg} / \mathrm{m}^{2}$ & $21.0(18.6-23.5)$ & $20.3(18.3-21.7)$ & 0.34 \\
\hline NYHA III or IV, n (\%) & $27(52)$ & $37(70)$ & 0.059 \\
\hline Systolic BP, mmHg & $129(108-149)$ & $126(101-139)$ & 0.26 \\
\hline Systolic BP >140 mmHg, n (\%) & $20(38)$ & $11(20)$ & 0.045 \\
\hline CS class $1 / 2 / 3, \%$ & $38 / 50 / 11$ & $20 / 60 / 19$ & 0.11 \\
\hline Diastolic BP, mmHg & $74(60-86)$ & $75(62-87)$ & 0.89 \\
\hline Heart rate, beats/min & $82(74-91)$ & $85(70-100)$ & 0.49 \\
\hline Atrial fibrillation, $\mathrm{n}(\%)$ & $12(23)$ & $19(36)$ & 0.15 \\
\hline Previous HF hospitalization, $\mathrm{n}(\%)$ & $27(52)$ & $35(66)$ & 0.14 \\
\hline \multicolumn{4}{|l|}{ Etiology } \\
\hline ICM, n (\%) & $21(40)$ & $18(34)$ & 0.49 \\
\hline DCM, n (\%) & $9(17)$ & $12(22)$ & 0.49 \\
\hline HCM, n (\%) & $2(4)$ & $3(6)$ & 0.66 \\
\hline HHD, n (\%) & $3(6)$ & $1(2)$ & 0.28 \\
\hline VHD, n (\%) & $8(15)$ & $5(9)$ & 0.35 \\
\hline HFpEF, n (\%) & $4(8)$ & $7(13)$ & 0.35 \\
\hline Other, n (\%) & $5(10)$ & $7(13)$ & 0.56 \\
\hline \multicolumn{4}{|l|}{ Medications } \\
\hline Inotropic drug infusion, $\mathrm{n}(\%)$ & $2(3)$ & $13(24)$ & 0.001 \\
\hline$\beta$-blocker, $\mathrm{n}(\%)$ & $27(52)$ & $29(55)$ & 0.77 \\
\hline ACEI or ARB, $n(\%)$ & $31(59)$ & $21(39)$ & 0.039 \\
\hline Diuretics, $n(\%)$ & $47(90)$ & $50(94)$ & 0.44 \\
\hline Dose of loop diuretics (furosemide-equivalent dose), mg & $40(40-40)$ & $40(40-80)$ & 0.005 \\
\hline \multicolumn{4}{|l|}{ Laboratory data } \\
\hline NT-proBNP, pg/mL & $5,175(2,586-11,1695)$ & $5,432(2,338-11,527)$ & 0.052 \\
\hline Hemoglobin, g/dL & $11.4(9.5-13.7)$ & $11.4(9.3-13.8)$ & 0.78 \\
\hline Platelet, $\times 10^{3} / \mu \mathrm{L}$ & 209 (158-267) & $165(123-208)$ & 0.012 \\
\hline Total bilirubin, $\mathrm{mg} / \mathrm{dL}$ & $0.62(0.43-0.94)$ & $0.98(0.51-1.86)$ & 0.005 \\
\hline AST, mg/dL & $27(21-41)$ & $30(23-47)$ & 0.28 \\
\hline $\mathrm{ALT}, \mathrm{mg} / \mathrm{dL}$ & $18(11-36)$ & $21(13-44)$ & 0.23 \\
\hline GGT, U/L & $22(16-43)$ & $65(31-157)$ & $<0.001$ \\
\hline Total cholesterol, mg/dL & $160(143-190)$ & 147 (124-159) & 0.003 \\
\hline Albumin, g/dL & $3.4(2.8-3.7)$ & $3.4(2.9-3.7)$ & 0.94 \\
\hline Sodium, mEq/L & $140(138-143)$ & $140(137-142)$ & 0.33 \\
\hline BUN, mg/dL & $21(14-30)$ & $29(18-47)$ & 0.014 \\
\hline Creatinine, $\mathrm{mg} / \mathrm{dL}$ & $1.0(0.7-1.5)$ & $1.3(0.8-2.0)$ & 0.078 \\
\hline eGFR, $\mathrm{mL} / \mathrm{min} / 1.73 \mathrm{~m}^{2}$ & $53(34-71)$ & $41(23-63)$ & 0.16 \\
\hline MELD-XI & $6.9(4.1-13.2)$ & $12.5(7.1-18.6)$ & 0.001 \\
\hline
\end{tabular}

Values are expressed as median (interquartile range), or $\mathrm{n}(\%)$. ACEI, angiotensin-converting enzyme inhibitor; ADHF, acute decompensated heart failure; ALT, alanine aminotransferase; ARB, angiotensin-receptor blocker; AST, aspartate aminotransferase; BP, blood pressure; BUN, blood urea nitrogen; CS, Clinical Scenarios; DCM, dilated cardiomyopathy; eGFR, estimated glomerular filtration rate; GGT, $\gamma$-glutamyltransferase; HCM, hypertrophic cardiomyopathy; HFpEF, heart failure with preserved ejection fraction; HHD, hypertensive heart disease; ICM, ischemic cardiomyopathy; LS, liver stiffness; MELD-XI, Model for End-stage Liver Disease excluding INR; NYHA, New York Heart Association; NT-proBNP, N-terminal pro-B-type natriuretic peptide; VHD, valvular heart disease.

\section{Follow-up}

All patients were followed from admission. Follow-up data were collected from medical records in a blinded manner. The primary endpoints were death from cardiovascular disease and readmission for HF.

\section{Statistical Analysis}

Continuous variables are expressed as median (interquartile range [IQR]) and compared by Mann-Whitney test. Cate- gorical variables are expressed as a number and percentage of patients and compared by chi-squared test. To satisfy the model assumptions, natural $\log$ transformation (ln) was used. Event-free survival was estimated by the KaplanMeier method, with group differences compared by logrank test. Univariate analysis based on a Cox proportional hazards model was used to evaluate the effects of $\ln$ LS (continuous variable) and high LS (above the median value, categorical variable) on cardiac events (death from 

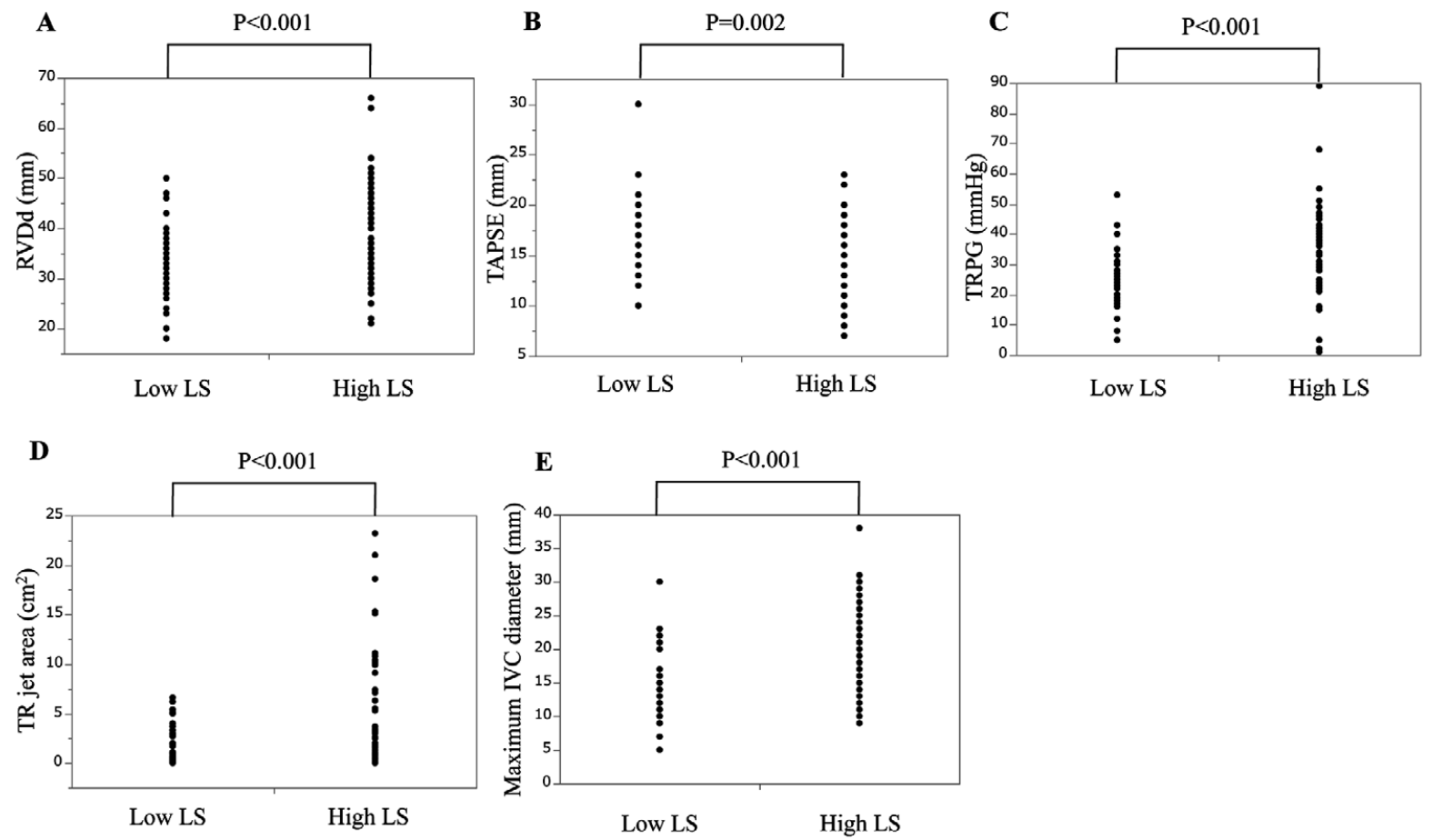

Figure 2. Distribution of right ventricular end-diastolic diameter (RVDd) (A), tricuspid annular plane systolic excursion (TAPSE) (B), tricuspid regurgitation pressure gradient (TRPG) (C), TR jet area (D), and maximum inferior vena cava (IVC) diameter (E) in patients stratified by median liver stiffness (LS): low LS <8.8 kPa [ $n=52]$ vs. high LS $\geq 8.8 \mathrm{kPa}$ [ $n=53$ ].

cardiovascular disease and readmission for $\mathrm{HF}$ ), and hazard ratios (HRs) with 95\% confidence intervals (CIs) were calculated. A multivariate model was used to adjust for the effect of variables related to organ congestion $(\ln [\mathrm{N}$-terminal pro-B-type natriuretic peptide (NT-proBNP)], estimated glomerular filtration rate (eGFR), total bilirubin, $\gamma$-glutamyl transferase (GGT), estimated RAP). Statistical analyses were conducted with JMP ver.11.0 (SAS Institute, Cary, $\mathrm{NC}$, USA). $\mathrm{P}<0.05$ was considered to be significant.

\section{Results}

\section{Patients' Characteristics}

The median (IQR) LS was 8.8 (4.9-21.6) $\mathrm{kPa}$. The clinical characteristics of patients divided into 2 groups according to the median LS (i.e., low LS $<8.8 \mathrm{kPa}$ and high LS $\geq 8.8 \mathrm{kPa}$ ) are shown in Table 1. Age, sex, BP, prevalence of atrial fibrillation, and cause of HF did not differ significantly between these groups. The high LS group had higher doses of diuretics [40 (40-80) vs. 40 [40-40] mg] and inotropic drug infusion $(24 \%$ vs. $3 \%)$ compared with the low LS group (both $\mathrm{P}<0.05$ ). The high LS group also had higher total bilirubin [0.98 $(0.51-1.86)$ vs. $0.62(0.43-0.94) \mathrm{mg} / \mathrm{dL}]$, GGT [65 (31-157) vs. 22 (16-43) mg/dL], and MELD-XI score $[12.5(7.1-18.6)$ vs. $6.9(4.1-13.2)]$, and lower total cholesterol [147 (124-159) vs. $160(143-190) \mathrm{mg} / \mathrm{dL}]$ and platelet count $\left[165(123-208) \times 10^{3} / \mu \mathrm{L}\right.$ vs. $209(158-267) \times$ $\left.10^{3} / \mu \mathrm{L}\right]$ compared with the low LS group (all $\mathrm{P}<0.05$ ). In echocardiographic data, the high LS group had a larger IVC diameter [22 (15-25) vs. 14 (10-17) mm], larger RVDd [ 41 (34 47) vs. 33 (27-36) mm], higher estimated RAP [15
(3-15) vs. 3 (3-8) $\mathrm{mmHg}$, higher prevalence of severe TR ( $52 \%$ vs. $14 \%$ ), and a lower level of TAPSE [15 (13-17) vs. 17 (15-19) mm] (Figure 2). Although LVDd, LVEF and E/e' did not differ significantly between the 2 groups, the high LS group had larger LAd [47 (42-52) vs. 43 (40-48) $\mathrm{mm}$ ], higher E/A ratio [2.1 (1.0-2.8) vs. 0.8 (0.7-1.6)], and lower deceleration time of the $\mathrm{E}$ wave [144 (115-170) vs. 176 (144-222) ms] (Table 2).

The low LS group had a significantly higher prevalence of the vascular failure pattern compared with the high LS group [38\% vs. $20 \%$ ] (Table 1). Furthermore, patients with a vascular failure pattern $(n=31)$ had a significantly lower LS than those with a non-vascular failure pattern $(n=74)$ [6.1 (5.2-9.6) vs. $10.4(4.8-26.2) \mathrm{kPa}, \mathrm{P}=0.034]$.

\section{LS and Clinical Outcomes}

There were 42 (40\%) cardiac events: 11 deaths from cardiovascular disease and 31 readmissions for HF during a median (IQR) follow-up period of 153 (83-231) days. Kaplan-Meier analysis showed that the high LS group had a significantly higher rate of cardiac events $(\mathrm{P}=0.001$ by log-rank test) (Figure 3A). Readmission for HF occurred in 20 patients $(37 \%)$ in the high LS group and in $11(21 \%)$ in the low LS group ( $\mathrm{P}=0.022$ by log-rank test) (Figure 3B). Cardiovascular death occurred in 13 patients $(24 \%)$ in the high LS group and in $6(11 \%)$ in the low LS group $(\mathrm{P}=0.088$ by log-rank test). Univariate Cox regression analysis revealed that cardiac events were strongly associated with previous hospitalization for HF (HR: 4.76, 95\% CI: 2.24 11.73, $\mathrm{P}<0.001$ ), high LS $\geq 8.8 \mathrm{kPa}$ (HR: $2.71,95 \% \mathrm{CI}$ : $1.43-5.43, \mathrm{P}=0.001)$, and higher $\ln [\mathrm{LS}](\mathrm{HR}: 1.57,95 \% \mathrm{CI}$ : 


\begin{tabular}{|c|c|c|c|}
\hline Item & $\begin{array}{l}\text { Low LS (<median) } \\
\qquad(n=52)\end{array}$ & $\begin{array}{c}\text { High LS ( } \geq \text { median) } \\
(n=53)\end{array}$ & $P$ value \\
\hline LVDd, mm & $54(47-63)$ & $55(48-60)$ & 0.93 \\
\hline LVDs, mm & $42(34-52)$ & $43(34-52)$ & 0.76 \\
\hline LVEF, \% & $38(30-56)$ & $41(29-53)$ & 0.88 \\
\hline LAd, mm & $43(40-48)$ & $47(42-52)$ & 0.009 \\
\hline $\mathrm{E}, \mathrm{ms}$ & $78(60-94)$ & $100(77-119)$ & 0.002 \\
\hline $\mathrm{A}, \mathrm{ms}$ & $80(54-95)$ & $42(32-79)$ & 0.005 \\
\hline $\mathrm{E} / \mathrm{A}$ ratio & $0.8(0.7-1.6)$ & $2.1(1.0-2.8)$ & 0.001 \\
\hline Deceleration time of $E$ wave, $\mathrm{ms}$ & $176(144-222)$ & $144(115-170)$ & 0.006 \\
\hline e' velocity, $\mathrm{cm} / \mathrm{s}$ & $4.4(3.5-6.0)$ & $5.0(4.0-7.0)$ & 0.10 \\
\hline a' velocity, $\mathrm{cm} / \mathrm{s}$ & $5.8(4.2-6.7)$ & $3.8(2.8-5.8)$ & 0.043 \\
\hline E/e' ratio & $15.3(11.8-18.6)$ & $15.6(13.2-21.7)$ & 0.26 \\
\hline RVDd, mm & $33(27-36)$ & $41(34-47)$ & $<0.001$ \\
\hline TAPSE, mm & $17(15-19)$ & $15(13-17)$ & 0.002 \\
\hline TRPG, $\mathrm{mmHg}$ & $23(16-30)$ & $34(24-41)$ & $<0.001$ \\
\hline MR III/IV, n (\%) & $14(29)$ & $18(36)$ & 0.47 \\
\hline TR III/IV, n (\%) & $7(14)$ & $26(52)$ & $<0.001$ \\
\hline TR jet area, $\mathrm{cm}^{2}$ & $0.6(0.2-2.8)$ & $3.1(1.2-7.8)$ & $<0.001$ \\
\hline Maximum IVC diameter, $\mathrm{mm}$ & $14(10-17)$ & $22(15-25)$ & $<0.001$ \\
\hline Estimated RAP, $\mathrm{mmHg}$ & $3(3-8)$ & $15(3-15)$ & $<0.001$ \\
\hline
\end{tabular}

Values are expressed as median (interquartile range), or $n(\%)$. A, late transmitral flow velocity; a', septal mitral annular late diastolic velocity; E, early transmitral flow velocity; e', septal mitral annular early diastolic velocity; E/e', ratio of peak mitral $E$ wave velocity to peak early diastolic myocardial velocity at septal position recorded by tissue Doppler imaging; IVC, inferior vena cava; LAd, left atrial diameter; LVDd, left ventricular end-diastolic diameter; LVDs, left ventricular end-systolic diameter; LVEF, left ventricular ejection fraction; MR, mitral regurgitation; RAP, right atrial pressure; RVDd, right ventricular end-diastolic diameter; TAPSE, tricuspid annular plane systolic excursion; TR, tricuspid regurgitation; TRPG, tricuspid regurgitation pressure gradient.

1.11-2.21, $\mathrm{P}=0.010$ ), aspartate aminotransferase (AST) (HR: $1.00,95 \%$ CI: $1.00-1.00, \mathrm{P}<0.001$ ), alanine aminotransferase (ALT) (HR: 1.00, 95\% CI: 1.00-1.00, $\mathrm{P}=0.001)$, blood urea nitrogen (BUN) (HR: 1.02, 95\% CI: 1.01-1.04, $\mathrm{P}<0.001$ ), and MELD-XI score (HR: $1.08,95 \%$ CI: $1.03-$ 1.13, $\mathrm{P}<0.001$ ) (Table 3). The significant association of high LS $\geq 8.8 \mathrm{kPa}$ with the incidence of death from cardiovascular disease and readmission for HF persisted in models adjusted for age, sex, and indices related to organ congestion (ln [NT-proBNP], BUN, eGFR, total bilirubin, GGT, estimated RAP) (Table 4).

\section{Discussion}

There were 2 major findings in this study. First, ADHF patients with higher LS had more elevated RAP (as assessed by echocardiography), hepatic congestion (total bilirubin, GGT), impaired hepatic synthesis (total cholesterol), and hepatic dysfunction (MELD-XI score). Second, high LS values were strongly associated with worse clinical outcomes, independent of other indices of organ congestion.

TE measures LS based on the velocity of an elastic shear wave that propagates in the liver, ${ }^{14}$ and LS measured by TE correlates strongly with the degree of fibrosis in liver biopsy obtained in chronic liver disease. ${ }^{15} \mathrm{TE}$ is a rapid, noninvasive and highly reproducible method. ${ }^{\mathbf{1 4}, 15}$ Because the liver is wrapped in a non-elastic capsule, liver congestion also leads to increased LS. ${ }^{6}$ Therefore, LS could be a marker of hepatic congestion or right-sided filling pressure in patients with general HF. ${ }^{7}$ Several groups have examined the effect of HF on the liver. ${ }^{16-18}$ In a clinical study, LS measured by TE increased in patients with HF, and inter- estingly, the increase in LS was greater in patients with right-sided HF than in patients with stable left HF. ${ }^{16}$ A recent study showed that TE-derived LS may be a predictor of RV failure after LV assist device implantation, ${ }^{19}$ but the relationships of LS with underlying cardiac condition and outcomes are not fully understood in patients with ADHF.

In the current study, LS in ADHF patients was related to elevated RAP and TR severity, and negatively associated with TAPSE, as manifested by RV systolic function. Regarding parameters of left-sided HF, LS was related to both the LAd and E/A ratio, and negatively correlated with deceleration time. These findings suggest that LS in ADHF patients is also influenced by elevated LV filling pressure. In addition, the patients with high LS had higher doses of diuretics and inotropic drug infusion. In ADHF patients, left-sided HF often causes postcapillary pulmonary hypertension $(\mathrm{PH})$ via increased LV filling pressure or pulmonary venous pressure. ${ }^{20}$ Persistent $\mathrm{PH}$ causes higher right heart pressure and right heart volume overload, leading to elevated RAP. ${ }^{20}$ Our findings suggested that high LS in ADHF patients is a robust marker of severe RV failure caused by right heart pressure and overload from postcapillary PH.

Patients with high LS in the current study also had higher bilirubin and GGT levels, lower total cholesterol, and higher MELD-XI scores, compared with those with low LS. Generally, HF is thought to cause hepatic congestion via increased RAP, ${ }^{1}$ and liver dysfunction caused by hepatic congestion is referred to as congestive hepatopathy. Laboratory data for hepatic congestion mainly show cholestasis, 2,21 with increased serum GGT, alkaline phosphatase, and bilirubin levels. Chronic liver congestion also 


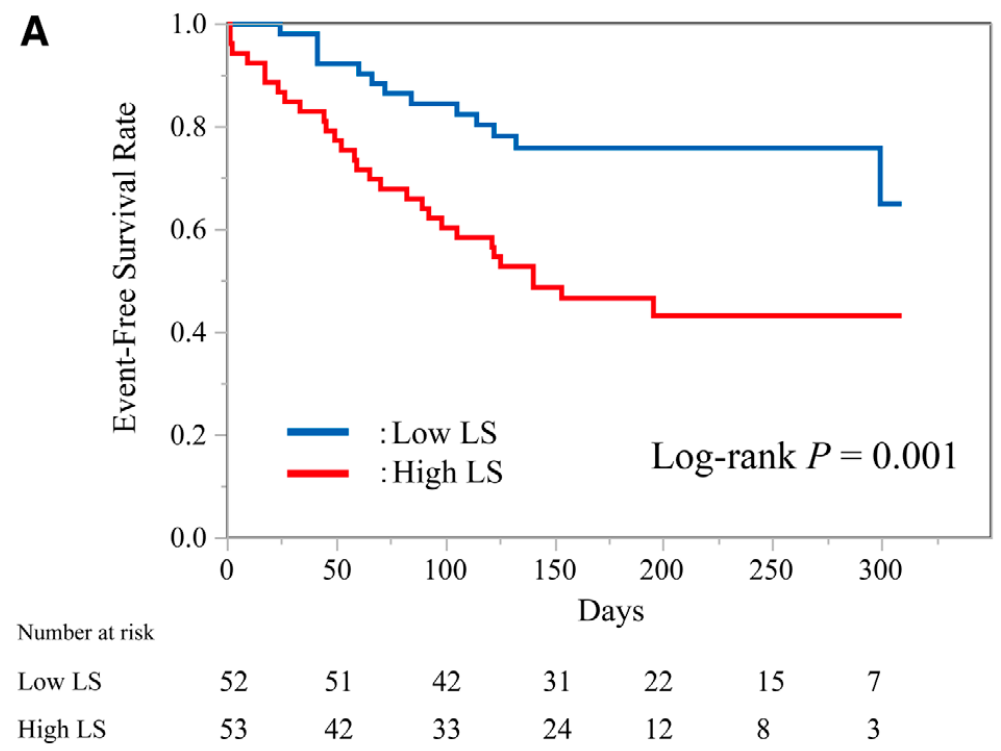

B

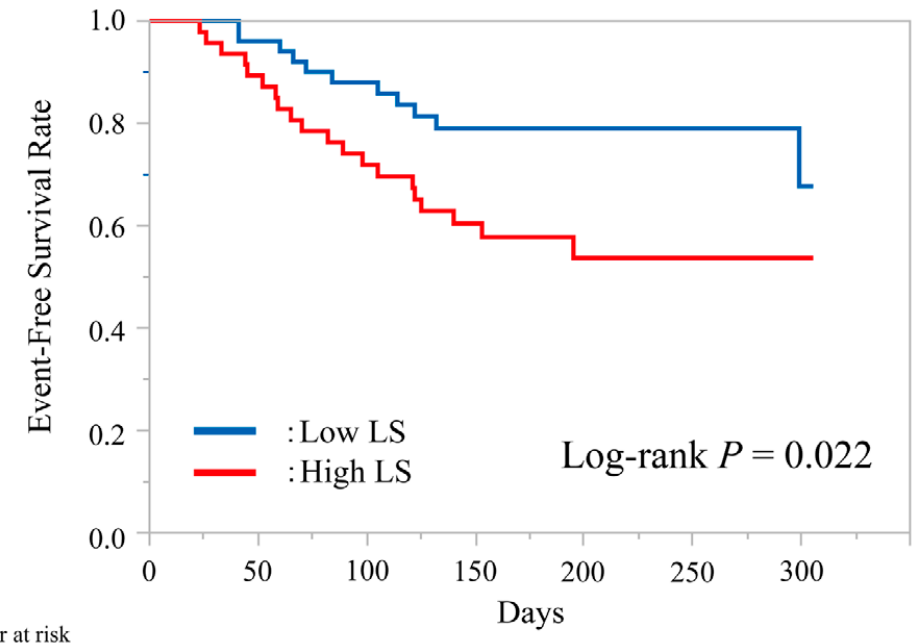

Number at risk

Low LS

$$
52 \quad 51
$$

High LS

51
42

$\begin{array}{lllll}42 & 31 & 22 & 15 & 7 \\ 33 & 24 & 12 & 8 & 3\end{array}$

Figure 3. Kaplan-Meier plot of event-free (cardiovascular death or readmission for heart failure) survival (A) and Kaplan-Meier plot of event-free (readmission for heart failure) survival (B), in patients in the high liver stiffness (LS) $(\geq 8.8 \mathrm{kPa}$, red line) and low LS (<8.8 kPa, blue line) groups. leads to liver damage and synthetic function impairment. Total cholesterol, albumin, and cholinesterase are markers of hepatic protein synthesis, ${ }^{22-24}$ while MELD-XI scores reflect liver damage caused by increased RAP.,13,25 Therefore, increased LS may reflect hepatic congestion induced by RV failure, impaired hepatic synthesis, and hepatic dysfunction.

Importantly, we showed that patients with ADHF with high LS ( $\geq$ median) had poor clinical outcomes. Furthermore, high LS was a predictor of clinical outcome after adjustment for laboratory and echocardiographic indices reflecting organ congestion, such as total bilirubin, GGT, and estimated RAP. To our knowledge, this is the first study to evaluate the prognostic significance of LS measured by TE in patients with ADHF. In fact, previous reports have shown the effect of liver congestion on the prognosis of HF. ${ }^{26-28}$ Total bilirubin correlates with hemodynamic variables, such as RAP, severity of TR, pulmonary wedge pressure, and cardiac output, ${ }^{26}$ and is a strong predictor of adverse outcomes in HF. ${ }^{27}$ In addition, the MELD and MELD-XI scores predict all-cause death in patients with advanced HF.,28 Our findings suggested that LS collectively reflects elevated RAP, liver congestion and liver injury, which are related to congestive hepatopathy, and thus, LS is an independent predictor of clinical outcome. TE is a simple, non-invasive, and user-friendly modality, and LS measured by TE may be useful for improving risk stratification and clinical management of patients with ADHF.

A previous study reported a cutoff value of LS of $10.6 \mathrm{kPa}$ for detection of RAP $>10 \mathrm{mmHg}{ }^{7}$ Compared with that value, a median LS value of $8.8 \mathrm{kPa}$ is low, but the patients in this study presented with ADHF. There may be 2 reasons for the low median LS. First, 39\% patients were in NYHA class II, and thus there is the possibility 


\begin{tabular}{|c|c|c|c|}
\hline Item & HR & $95 \% \mathrm{Cl}$ & $P$ value \\
\hline Age (per 1year increase) & 1.01 & $0.98-1.03$ & 0.33 \\
\hline Sex male & 0.90 & $0.47-1.84$ & 0.78 \\
\hline Systolic BP (per $1 \mathrm{mmHg}$ increase) & 0.99 & $0.98-1.00$ & 0.60 \\
\hline Atrial fibrillation & 1.57 & $0.81-2.92$ & 0.17 \\
\hline Previous HF hospitalization & 4.76 & $2.24-11.73$ & $<0.001$ \\
\hline In [NT-proBNP] (per 1.0 increase) & 1.16 & $0.89-1.53$ & 0.27 \\
\hline Total bilirubin (per $1 \mathrm{mg} / \mathrm{dL}$ increase) & 1.16 & $0.95-1.33$ & 0.11 \\
\hline AST (per $1 \mathrm{mg} / \mathrm{dL}$ increase) & 1.00 & $1.00-1.00$ & $<0.001$ \\
\hline ALT (per $1 \mathrm{mg} / \mathrm{dL}$ increase) & 1.00 & $1.00-1.00$ & 0.001 \\
\hline GGT (per 1U/L increase) & 0.99 & $0.99-1.00$ & 0.95 \\
\hline BUN (per $1 \mathrm{mg} / \mathrm{dL}$ increase) & 1.02 & $1.01-1.04$ & $<0.001$ \\
\hline eGFR (per $1 \mathrm{~mL} / \mathrm{min} / 1.73 \mathrm{~m}^{2}$ increase) & 0.98 & $0.96-0.99$ & 0.003 \\
\hline MELD-XI & 1.08 & $1.03-1.13$ & $<0.001$ \\
\hline LVDd (per $1 \mathrm{~mm}$ increase) & 0.97 & $0.94-1.00$ & 0.13 \\
\hline Reduced LVEF (LVEF <50\%) & 0.52 & $0.27-1.03$ & 0.061 \\
\hline RVDd (per $1 \mathrm{~mm}$ increase) & 1.04 & $1.00-1.07$ & 0.034 \\
\hline Estimated RAP (per $1 \mathrm{mmHg}$ increase) & 1.06 & $0.99-1.12$ & 0.057 \\
\hline TRPG (per $1 \mathrm{mmHg}$ increase) & 1.01 & $0.99-1.03$ & 0.087 \\
\hline TR jet area (per $1 \mathrm{~cm}^{2}$ increase) & 1.03 & $0.97-1.08$ & 0.18 \\
\hline TAPSE (per $1 \mathrm{~mm}$ increase) & 0.91 & $0.83-1.00$ & 0.065 \\
\hline In [LS] (per 1.0 increase) & 1.57 & $1.11-2.21$ & 0.010 \\
\hline High LS ( $\geq$ median) & 2.71 & $1.43-5.43$ & 0.001 \\
\hline
\end{tabular}

${ }^{*}$ Cardiac event defined as death from cardiovascular disease or readmission for heart failure. $\mathrm{Cl}$, confidence interval; $\mathrm{HR}$, hazard ratio; In, natural log transformation. Other abbreviations as in Tables 1,2.

\begin{tabular}{|c|c|c|c|c|c|c|}
\hline \multirow{2}{*}{ Model } & \multicolumn{3}{|c|}{ In [LS] (per 1.0 increase) } & \multicolumn{3}{|c|}{ High LS ( $\geq$ median) } \\
\hline & HR & $95 \% \mathrm{Cl}$ & $P$ value & HR & $95 \% \mathrm{Cl}$ & $P$ value \\
\hline 1 (age, sex, In [NT-proBNP]) & 1.71 & $1.18-2.50$ & 0.004 & 2.95 & $1.54-5.95$ & $<0.001$ \\
\hline 2 (age, sex, BUN) & 1.35 & $0.93-1.96$ & 0.10 & 2.42 & $1.24-4.98$ & 0.009 \\
\hline 3 (age, sex, eGFR) & 1.57 & $1.10-2.25$ & 0.011 & 2.89 & $1.50-5.89$ & 0.001 \\
\hline 4 (age, sex, total bilirubin) & 1.56 & $1.07-2.26$ & 0.019 & 2.76 & $1.41-5.66$ & 0.002 \\
\hline 5 (age, sex, GGT) & 1.55 & $1.07-2.23$ & 0.018 & 2.66 & $1.35-5.49$ & 0.004 \\
\hline 6 (age, sex, estimated RAP) & 1.63 & $0.94-2.84$ & 0.07 & 2.57 & $1.08-5.99$ & 0.032 \\
\hline
\end{tabular}

${ }^{*}$ Cardiac event defined as death from cardiovascular disease or readmission for heart failure. Abbreviations as in Tables 1-3.

that mild HF patients were included in the study. Second, 31 of 105 patients $(29 \%)$ presented with vascular failure pattern onset, defined as SBP $>140 \mathrm{mmHg}$ at admission, ${ }^{9}$ and patients with a vascular failure pattern had significantly lower LS than those with a non-vascular failure pattern. Furthermore, the low LS group had a significantly higher prevalence of a vascular failure pattern compared with the high LS group. This indicates that patients with low LS at admission tend to present with vascular failure and that organ congestion is not severe, compared with those with high LS.

\section{Study Limitations}

First, the underlying histology of liver fibrosis was not considered. However, no patients with liver dysfunction or chronic liver disease and none who consumed alcohol in excess (>20 g/day) were included in the study. Second, LS is technically difficult to obtain accurately by TE in patients who are obese, have a narrow intercostal space, or have substantial ascites. Third, LS data at discharge were not obtained, and a previous study showed that ADHF has a high in-hospital mortality rate. ${ }^{29} \mathrm{We}$ only collected LS data at admission because the aim of the study was to investigate prediction of prognosis in ADHF patients using LS values, and we evaluated the prognostic value of LS for cardiac events, including in-hospital death. However, LS data at discharge may help with management of HF patients. Further studies are needed to clarify this issue. Finally, the study was performed at a single center and included a relatively small number of patients. Within these limitations, we conclude that increased LS values reflect RAP elevation, hepatic congestion, and hepatic dysfunction, and that LS upon admission may be a useful prognostic marker for cardiac events in patients with ADHF.

\section{Disclosures}

We have no conflicts of interest to disclose. 


\section{Acknowledgments / Funding Sources}

None.

\section{References}

1. Allen LA, Felker GM, Pocock S, McMurray JJ, Pfeffer MA, Swedberg K, et al. Liver function abnormalities and outcome in patients with chronic heart failure: Data from the Candesartan in Heart Failure: Assessment of Reduction in Mortality and Morbidity (CHARM) program. Eur J Heart Fail 2009; 11: 170177.

2. Poelzl G, Ess M, Mussner-Seeber C, Pachinger O, Frick M, Ulmer H. Liver dysfunction in chronic heart failure: Prevalence, characteristics and prognostic significance. Eur J Clin Invest 2012; 42: 153-163.

3. Kim MS, Kato TS, Farr M, Wu C, Givens RC, Collado E, et al. Hepatic dysfunction in ambulatory patients with heart failure: Application of the MELD scoring system for outcome prediction. $J$ Am Coll Cardiol 2013; 61: 2253-2261.

4. Chon YE, Choi EH, Song KJ, Park JY, Kim DY, Han KH, et al. Performance of transient elastography for the staging of liver fibrosis in patients with chronic hepatitis B: A meta-analysis. PLoS One 2012; 7: e44930.

5. Gaia S, Carenzi S, Barilli AL, Bugianesi E, Smedile A, Brunello F, et al. Reliability of transient elastography for the detection of fibrosis in non-alcoholic fatty liver disease and chronic viral hepatitis. J Hepatol 2011; 54: 64-71.

6. Millonig G, Friedrich S, Adolf S, Fonouni H, Golriz M, Mehrabi A, et al. Liver stiffness is directly influenced by central venous pressure. J Hepatol 2010; 52: 206-210.

7. Taniguchi T, Sakata Y, Ohtani T, Mizote I, Takeda Y, Asano Y, et al. Usefulness of transient elastography for noninvasive and reliable estimation of right-sided filling pressure in heart failure. Am J Cardiol 2014; 113: 552-558.

8. McKee PA, Castelli WP, McNamara PM, Kannel WB. The natural history of congestive heart failure: The Framingham study. N Engl J Med 1971; 285: 1441-1446.

9. Mebazaa A, Gheorghiade M, Pina IL, Harjola VP, Hollenberg SM, Follath F, et al. Practical recommendations for prehospital and early in-hospital management of patients presenting with acute heart failure syndromes. Crit Care Med 2008; 36: S129S139.

10. Castera L, Forns X, Alberti A. Non-invasive evaluation of liver fibrosis using transient elastography. J Hepatol 2008; 48: 835847.

11. Lang RM, Bierig M, Devereux RB, Flachskampf FA, Foster E, Pellikka PA, et al. Recommendations for chamber quantification: A report from the American Society of Echocardiography's Guidelines and Standards Committee and the Chamber Quantification Writing Group, developed in conjunction with the European Association of Echocardiography, a branch of the European Society of Cardiology. J Am Soc Echocardiogr 2005; 18: $1440-1463$.

12. Rudski LG, Lai WW, Afilalo J, Hua L, Handschumacher MD, Chandrasekaran K, et al. Guidelines for the echocardiographic assessment of the right heart in adults: A report from the American Society of Echocardiography endorsed by the European Association of Echocardiography, a registered branch of the European Society of Cardiology, and the Canadian Society of Echocardiography. J Am Soc Echocardiogr 2010; 23: 685-713.

13. Abe S, Yoshihisa A, Takiguchi M, Shimizu T, Nakamura Y, Yamauchi H, et al. Liver dysfunction assessed by model for end-stage liver disease excluding INR (MELD-XI) scoring system predicts adverse prognosis in heart failure. PLoS One
2014; 9: e100618.

14. Martinez SM, Crespo G, Navasa M, Forns X. Noninvasive assessment of liver fibrosis. Hepatology 2011; 53: 325-335.

15. Castera L, Vergniol J, Foucher J, Le Bail B, Chanteloup E, Haaser M, et al. Prospective comparison of transient elastography, Fibrotest, APRI, and liver biopsy for the assessment of fibrosis in chronic hepatitis C. Gastroenterology 2005; 128: 343-350.

16. Hopper I, Kemp W, Porapakkham P, Sata Y, Condon E, Skiba $\mathrm{M}$, et al. Impact of heart failure and changes to volume status on liver stiffness: Non-invasive assessment using transient elastography. Eur J Heart Fail 2012; 14: 621 - 627.

17. Chon YE, Kim SU, Park JY, Kim DY, Ahn SH, Han KH, et al. Dynamics of the liver stiffness value using transient elastography during the perioperative period in patients with valvular heart disease. PLoS One 2014; 9: e92795.

18. Colli A, Pozzoni P, Berzuini A, Gerosa A, Canovi C, Molteni $\mathrm{EE}$, et al. Decompensated chronic heart failure: Increased liver stiffness measured by means of transient elastography. Radiology 2010; 257: 872-878.

19. Kashiyama N, Toda K, Nakamura T, Miyagawa S, Nishi H, Yoshikawa Y, et al. Evaluation of right ventricular function using liver stiffness in patients with left ventricular assist device. Eur J Cardiothorac Surg 2017; 51: 715-721.

20. Galie N, Hoeper MM, Humbert M, Torbicki A, Vachiery JL, Barbera JA, et al. Guidelines for the diagnosis and treatment of pulmonary hypertension: The Task Force for the Diagnosis and Treatment of Pulmonary Hypertension of the European Society of Cardiology (ESC) and the European Respiratory Society (ERS), endorsed by the International Society of Heart and Lung Transplantation (ISHLT). Eur Heart J 2009; 30: 2493-2537.

21. Lau GT, Tan HC, Kritharides L. Type of liver dysfunction in heart failure and its relation to the severity of tricuspid regurgitation. Am J Cardiol 2002; 90: 1405-1409.

22. Horwich TB, Hamilton MA, Maclellan WR, Fonarow GC. Low serum total cholesterol is associated with marked increase in mortality in advanced heart failure. J Card Fail 2002; 8: 216-224.

23. Liu M, Chan CP, Yan BP, Zhang Q, Lam YY, Li RJ, et al. Albumin levels predict survival in patients with heart failure and preserved ejection fraction. Eur J Heart Fail 2012; 14: 39-44.

24. Sato T, Yamauchi H, Suzuki S, Yoshihisa A, Yamaki T, Sugimoto K, et al. Serum cholinesterase is an important prognostic factor in chronic heart failure. Heart Vessels 2015; 30: 204-210.

25. Scholfield M, Schabath MB, Guglin M. Longitudinal trends, hemodynamic profiles, and prognostic value of abnormal liver function tests in patients with acute decompensated heart failure: An analysis of the ESCAPE trial. J Card Fail 2014; 20: 476-484.

26. Kubo SH, Walter BA, John DH, Clark M, Cody RJ. Liver function abnormalities in chronic heart failure: Influence of systemic hemodynamics. Arch Intern Med 1987; 147: 1227-1230.

27. Shinagawa $H$, Inomata $T$, Koitabashi $T$, Nakano H, Takeuchi I, Naruke T, et al. Prognostic significance of increased serum bilirubin levels coincident with cardiac decompensation in chronic heart failure. Circ J 2008; 72: 364-369.

28. Yang JA, Kato TS, Shulman BP, Takayama H, Farr M, Jorde UP, et al. Liver dysfunction as a predictor of outcomes in patients with advanced heart failure requiring ventricular assist device support: Use of the Model of End-stage Liver Disease (MELD) and MELD eXcluding INR (MELD-XI) scoring system. $J$ Heart Lung Transplant 2012; 31: 601-610.

29. Fonarow GC, Adams KF Jr, Abraham WT, Yancy CW, Boscardin WJ. Risk stratification for in-hospital mortality in acutely decompensated heart failure: Classification and regression tree analysis. JAMA 2005; 293: $572-580$. 